\title{
Frecuencia de tromboembolismo venoso en pacientes hospitalizados con cáncer. Factores de riesgo y eficacia de la tromboprofilaxis farmacológica
}

\author{
G uillermo Conte L, G astón Figueroa M. \\ Frequency of venous \\ thromboembolism among \\ hospitalized patients with cancer
}

\begin{abstract}
Background: Hospitalized patients with cancer have a high risk of venous thromboembolism (VTE). Aim: To study the frequency of VTE and its risk factors in hospitalized patients with cancer. Material and methods: Retrospective analysis of clinical records of patients with cancer, hospitalized at a university hospital between 2002 and 2004 . Patients with the diagnosis of VTE at admission or using anticoagulants, were excluded from the analysis. Results: The medical records of 366 patients were reviewed. Fifty three percent had a digestive cancer, 19\% lung cancer, $10 \%$ breast cancer and $18 \%$ had a tumor of other origin. In $77 \%$, the tumor was in an advanced stage. The most common admission diagnoses were pneumonia, vomiting and dehydration, gastrointestinal bleeding and urinary infection. In 125 patients (34\%) pharmacological thrombo-prophylaxis was not used and 242 (66\%) received regular or low molecular weight heparin. VTE was detected in 11 patients (3\%) and was significantly more common among patients not receiving thrombo prophylaxis compared to those receiving heparin $(6.4 \%$ and $1.2 \%$, respectively, $p=0.014)$. Factors associated to VTE were a history of previous VTE with an odds ratio (OR) of $12.9(\mathrm{p}<0.01)$, obesity with an OR of 13.3 ( $p<0.01$ ), recent chemotherapy with an OR of 6.9 ( $p=0.01$ ). The use of pharmacological thromboprophylaxis had an OR of $0.24(p=0.05)$. Conclusions: Three percent of patients in this series had VTE during the hospitalization. Pharmacological thrombo-prophylaxis significantly reduced the risk of VTE (Rev Méd Chile 2008; 136: 1528-34).
\end{abstract}

(Key words: Fibrinolytic agents; Neoplasms; Venous thromboembolism)

Recibido el 23 de junio, 2008. Aceptado el 2 de septiembre, 2008.

Fuentes de financiamiento: Financiamiento parcial por parte de Laboratorio Pfizer. Esta organización no tuvo participación en el diseño del estudio, en la recolección, análisis o interpretación de los datos, ni en la preparación y revisión del manuscrito.

Sección de Hematología, Hospital Clínico Universidad de Chile. Santiago de Chile.

$\overline{\text { Correspondencia a: Dr. Guillermo Conte L. Sección de }}$ Hematología, Hospital Clínico Universidad de Chile. Santos Dumont 999, Independencia, Santiago, Chile. Fax: 7777618. E mail: gfconte@gmail.com 
$\mathrm{E}^{1}$ tromboembolismo venoso (TEV), que incluye a la trombosis venosa profunda y el tromboembolismo pulmonar, es una condición grave y potencialmente fatal que puede complicar la evolución de pacientes hospitalizados por cuadros médicos o quirúrgicos ${ }^{1}$. Dependiendo de las características de la población estudiada, se estima que puede afectar a 5\%-15\% de los pacientes hospitalizados ${ }^{1}$ y ser responsable de hasta $5 \%$ de las muertes que ocurren en el hospital ${ }^{2,3}$.

Un metaanálisis que incluyó 9 estudios y 19.958 pacientes demostró que la tromboprofilaxis farmacológica es una efectiva medida para disminuir la frecuencia de TEV en pacientes médicos ${ }^{4}$. Con el fin de obtener el máximo beneficio de la tromboprofilaxis farmacológica es necesario identificar poblaciones de alto riesgo de TEV. Estudios prospectivos realizados en pacientes médicos han identificado diversos factores de riesgo de TEV entre los cuales destaca el diagnóstico de cáncer ${ }^{5}$.

Los objetivos de este estudio son describir la frecuencia de TEV sintomático en una población de pacientes con cáncer hospitalizados en un centro universitario por cuadros médicos agudos, identificar factores de riesgo y analizar la eficacia de la tromboprofilaxis farmacológica.

\section{PACIENTES Y MÉTODOS}

Estudio descriptivo y retrospectivo, en el cual se analizaron los registros clínicos de pacientes ingresados al Hospital Clínico de la Universidad de Chile durante el periodo comprendido entre enero de 2002 a diciembre de 2004. Los pacientes se seleccionaron a través de la información contenida en los registros de altas médicas de la Oficina de Estadística de este centro. Los criterios de inclusión fueron: diagnóstico de cáncer confirmado con estudio histopatológico, motivo de hospitalización no relacionado al tratamiento del cáncer (cirugía, quimioterapia) y estadía hospitalaria superior a cuatro días. Los criterios de exclusión fueron: edad menor a 18 años, ingreso a Unidad de Cuidados Intensivos o Unidad Coronaria, diagnóstico de TEV al ingreso y uso de dosis terapéuticas de anticoagulantes orales (cumarínicos), heparina no fraccionada o heparinas de bajo peso molecular al momento del ingreso.

Las variables clínicas y de laboratorio registradas al ingreso fueron: edad, sexo, diagnóstico de ingreso, localización y estadio (TNM) del cáncer, creatininemia, protrombinemia y recuento de plaquetas.

Se registró la presencia de los siguientes factores de riesgo de TEV identificados en estudios prospectivos ${ }^{4,5}$ : edad mayor a 70 años, infección activa, insuficiencia cardiaca descompensada, antecedentes de TEV, obesidad (Índice de masa corporal (IMC) >30), diagnóstico de trombofilia, cirugía oncológica reciente (<30 días), quimioterapia (QMT) reciente ( $<30$ días).

Las variables registradas durante la hospitalización fueron: uso de tromboprofilaxis farmacológica (droga, dosis, día de inicio), eventos TEV diagnosticados por métodos objetivos (eco-doppler venoso o angio-TAC de tórax), eventos hemorrágicos mayores (definidos como aquellos que requieren intervención quirúrgica, procedimiento endoscópico o transfusión de glóbulos rojos) y estado al alta (vivo o fallecido).

Las variables continuas se describen como mediana y rango y las categoriales como porcentajes. El impacto de todas la variables registradas sobre la frecuencia de TEV fue analizado en primer lugar por análisis univariable (chi cuadrado). Para analizar la eficacia de la tromboprofilaxis farmacológica se realizó un análisis de regresión logística multivariable en el que se incluyó el uso de tromboprofilaxis y todas las variables clínicas con $\mathrm{p}<0,2$ identificadas en el análisis univariable. Para los análisis estadísticos se utilizó el programa SPSS 13.0 (SPSS Inc. Chicago, Illinois, USA).

\section{Resultados}

Durante el periodo estudiado se identificaron 366 pacientes que cumplían los criterios de inclusión y exclusión. La mediana de edad de los pacientes fue de 63 años (rango 29-85). Las características clínicas y exámenes de laboratorio registrados al ingreso de los pacientes se presentan en la Tabla 1. De los exámenes de laboratorio destaca una baja frecuencia de falla renal $(2,7 \%)$ y trombocitopenia $(14,7 \%)$. La protrombinemia fue superior a $40 \%$ en todos los casos. Respecto a las neoplasias las localizaciones más frecuentes fueron estómago (38\%), pulmón $(18,9 \%)$, colon $(15,3 \%)$, mama $(9,8 \%)$. La enfermedad neoplásica se encontraba 
Tabla 1. C aracterísticas clínicas, hallazgos de laboratorio, motivo de ingreso y localización de neoplasia en pacientes con cáncer hospitalizados por cuadros médicos agudos

\begin{tabular}{|c|c|c|}
\hline & $\mathbf{n}$ & $\%$ \\
\hline Total de pacientes & 366 & $100 \%$ \\
\hline Hombre/mujer & $161 / 205$ & $44 / 56$ \\
\hline Edad mediana (rango) & $63(29-85)$ & \\
\hline \multicolumn{3}{|l|}{ Laboratorio } \\
\hline Creatininemia $>2 \mathrm{mg} / \mathrm{dL}$ & 10 & 2,7 \\
\hline Protrombina <40\% & 0 & 0 \\
\hline Trombocitopenia $(<150.000 / \mu \mathrm{L})$ & 54 & 14,7 \\
\hline \multicolumn{3}{|l|}{ Motivo de Ingreso* } \\
\hline Neumonía & 63 & 17,2 \\
\hline Síndrome emético/deshidratación & 61 & 16,7 \\
\hline Hemorragia digestiva & 25 & 9,1 \\
\hline Infección urinaria & 27 & 7,4 \\
\hline Diabetes descompensada & 27 & 7,4 \\
\hline EPOC descompensado & 22 & 6,0 \\
\hline Obstrucción intestinal & 16 & 4,6 \\
\hline Colangitis & 15 & 4,4 \\
\hline \multicolumn{3}{|l|}{ Localización del cáncer } \\
\hline Estómago & 139 & 38,0 \\
\hline Pulmón & 69 & 18,9 \\
\hline Colon & 56 & 15,3 \\
\hline Mama & 36 & 9,8 \\
\hline Hígado & 18 & 4,9 \\
\hline Otro & 46 & 13,1 \\
\hline
\end{tabular}

EPOC: enfermedad pulmonar obstructiva crónica.

*Se listan los motivos de ingresos con más de 10 casos.

en un estadio avanzado (TNM 3-4) en 281 (76,7\%) de los casos. La mediana de estadía hospitalaria fue de 9 días (rango 4-57) y 35 pacientes $(9,5 \%)$ fallecieron durante la hospitalización. La frecuencia de diferentes factores de riesgo de TEV se detalla en la Tabla 2. En 129 pacientes (35,2\%) no se identificaron factores de riesgo de TEV, 148 pacientes $(40,4 \%)$ presentaban 1 factor de riesgo y 89 pacientes $(24,3 \%)$ presentaban 2 ó más de los factores de riesgo detallados en la Tabla 2.

Del total de pacientes, 125 (34,2\%) no recibió ninguna forma de tromboprofilaxis farmacológica, 120 pacientes $(32,8 \%)$ recibieron heparina no fraccionada por vía subcutánea (5.000 U c/8 ó 12 h) y 121 pacientes $(33,1 \%)$ recibieron una heparina de bajo peso molecular (dalteparina o enoxaparina). En 200 pacientes (54\%) se indicó la utilización de medias antitrombóticas, sin embargo la revisión de los registros clínicos no pudo confirmar su utilización en más de la mitad de los casos. En ningún caso se utilizó compresión neumática intermitente.

La proporción de pacientes que recibieron tromboprofilaxis no fue afectada por el número de factores de riesgo identificados al ingreso $(0$ factor: $77,5 \%$; 1 factor: $60,1 \%$; $>1$ factor: $70,7 \%$ ).

Durante la hospitalización 11 pacientes (3\%) presentaron 11 eventos de TEV ( 8 casos de trombosis venosa profunda y 3 casos de tromboembolismo pulmonar). La frecuencia de TEV en los pacientes que no recibieron tromboprofilaxis farmacológica fue de $6,4 \%(8 / 125)$, que fue significativamente superior al 1,2\% (3/241) observado en los pacientes que recibieron tromboprofilaxis farmacológica $(p=0,014)$. 
Tabla 2. Frecuencia de factores de riesgo de T EV $(n=366)$

\begin{tabular}{|lrr|}
\hline & $\mathbf{n}$ & $\%$ \\
\hline Infección activa & 129 & 35,2 \\
Edad >70 años & 98 & 26,8 \\
Cirugía oncológica reciente & 51 & 13,9 \\
Quimioterapia reciente & 45 & 12,3 \\
Obesidad (IMC >30) & 18 & 4,9 \\
Antecedentes de TEV & 15 & 4,1 \\
Insuficiencia cardiaca descompensada & 6 & 1,6 \\
Diagnóstico de trombofilia & 0 & 0 \\
\hline
\end{tabular}

IMC: índice de masa corporal

Para cuantificar el impacto de los diferentes factores de riesgo de TEV registrados en este estudio se realizó en primer lugar un análisis univariable del que resultaron significativos el antecedente de TEV, quimioterapia reciente y obesidad (Tabla 3). El análisis multivariable incluyendo estas tres variables y el uso de tromboprofilaxis farmacológica reveló que esta medida terapéutica disminuye significativamente el riesgo de TEV (OR 0,24; $\mathrm{p}=0,047$ ) (Tabla 3).

Para graficar de mejor forma la interacción entre estos factores de riesgo y la utilización de tromboprofilaxis, en la Figura 1 se grafica la frecuencia de TEV según el uso de tromboprofilaxis farmacológica y la presencia de alguno de los 3 factores de riesgo de TEV identificados por análisis multivariable. Este gráfico revela que el efecto protector del uso de tromboprofilaxis farmacológica es aún más notorio en el grupo de pacientes con 1 o más factores de riesgo (frecuencia TEV 20\% vs 2,8\%; OR 0,11 [IC 95\% 0,01-0,76]; p $=0,02$ ).

El uso de tromboprofilaxis no se asoció a un aumento en la frecuencia de complicaciones hemorrágicas mayores ( $\sin$ profilaxis $1,6 \%$; con profilaxis $2,0 \%$ ).

\section{Tabla 3. Análisis univariable y multivariable del efecto de diferentes factores de riesgo y el uso de tromboprofilaxis farmacológica en la incidencia de T EV en 366 pacientes médicos con cáncer}

\begin{tabular}{|lccc|}
\hline & $\begin{array}{c}\text { Análisis univariable* } \\
\text { Análisis multivariable** } \\
\text { O R }\end{array}$ & p \\
\hline Infección activa & $\mathbf{p}$ & & \\
Edad $>70$ años & $\mathrm{ns}$ & & \\
Cirugía oncológica reciente & $\mathrm{ns}$ & 6,9 & 0,011 \\
Quimioterapia reciente & $\mathrm{ns}$ & 13,3 & 0,008 \\
Obesidad (IMC $>30$ ) & 0,01 & 12,9 & 0,002 \\
Antecedentes de TEV & 0,04 & & \\
Insuficiencia cardiaca descompensada & $<0,001$ & 0,24 & 0,047 \\
TNM 3-4 & $\mathrm{ns}$ & & \\
Uso tromboprofilaxis & $\mathrm{ns}$ & & \\
\hline
\end{tabular}

*Chi cuadrado con corrección de Yates ( 2 colas); **Regresión logística multivariable: OR odds ratio. ns: no significativo (todos $>0,2)$ 


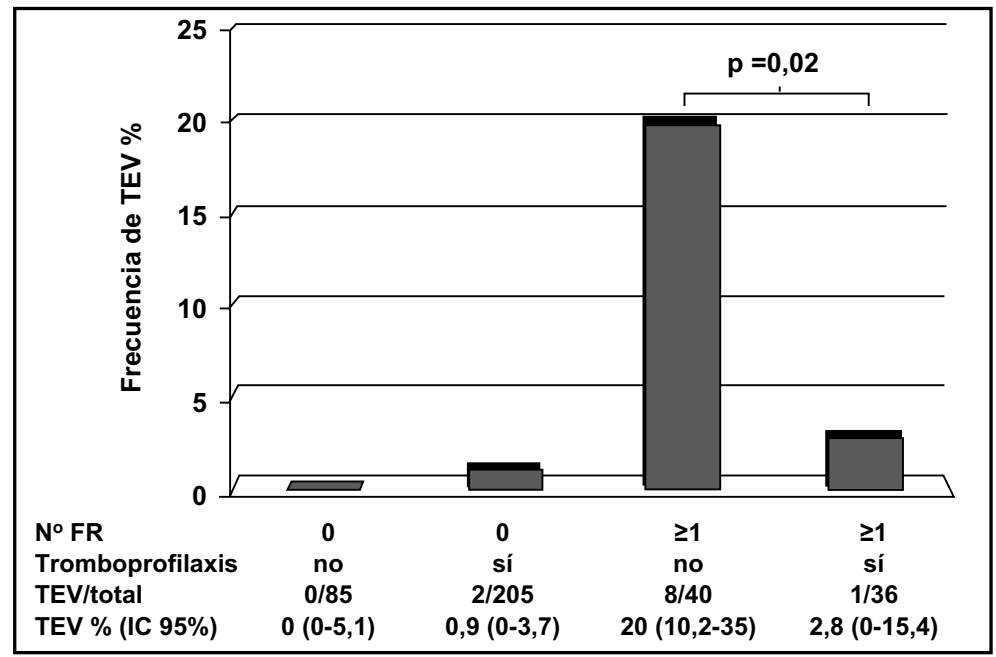

Figura 1. Frecuencia de tromboembolismo venoso (TEV) según el número de factores de riesgo y uso de tromboprofilaxis. Los factores de riesgo considerados fueron: antecedentes de TEV, quimioterapia reciente y obesidad (IMC >30). FR: Factores de riesgo. IC: Intervalo de confianza.

\section{DISCUSIÓN}

Por muchos años los estudios respecto al TEV en pacientes hospitalizados estuvieron centrados en la población de pacientes quirúrgicos. En los últimos años una serie de estudios revelaron que más de 50\% de los casos de TEV intrahospitalario ocurren en pacientes médicos y que algunos grupos de pacientes médicos pueden tener un riesgo sustancial de $\mathrm{TEV}^{6,7}$.

La eficacia de la tromboprofilaxis farmacológica en pacientes médicos ha sido demostrada en tres estudios prospectivos (MEDENOX, PREVENT, ARTEMIS) utilizando enoxaparina (40 mg al día), dalteparina (5.000 U al día) y fondaparinux (2,5 $\mathrm{mg}$ al día), respectivamente, con riesgos relativos observados de 0,37 a 0,55 (8-10). Por otro lado, el subanálisis de estos estudios y otros observacionales demostraron que el diagnóstico de cáncer es un importante factor de riesgo de TEV intrahospitalario 4,5 .

El presente estudio ha revelado que los pacientes con cáncer hospitalizados por causas médicas tienen un alto riesgo de TEV sintomático (3\% en esta serie) y que este riesgo puede disminuirse significativamente con el uso de tromboprofilaxis farmacológica. Otras series de pacien- tes con cáncer han informado una frecuencia de TEV sintomático de $2 \%$ a $8 \%{ }^{11-13}$. Aunque la tromboprofilaxis farmacológica ha sido extensamente estudiada en pacientes sometidos a cirugía oncológica $^{14}$, pocos estudios han evaluado su eficacia en pacientes médicos con cáncer. En un subanálisis del estudio MEDENOX la tromboprofilaxis con enoxaparina disminuyó significativamente el riesgo de TEV (OR 0,50; IC 95\% $0,14-0,72)^{15}$.

En nuestro estudio observamos una importante reducción en el riesgo de TEV con el uso de tromboprofilaxis farmacológica (1,2\% vs 6,4\%) y este efecto es más notorio en el grupo de pacientes con uno o más factores de riesgo de TEV (2,8\% vs 20\%). Los factores de riesgo identificados en este estudio (antecedentes de TEV, quimioterapia recientes y obesidad) son concordantes con los reportados en la literatura ${ }^{13,16}$. Entre éstos ha emergido la quimioterapia como un muy importante factor de riesgo de TEV tanto para pacientes hospitalizados como ambulato$\operatorname{rios}^{17-19}$.

Un hecho a destacar en nuestro estudio es la proporción de pacientes que no recibieron tromboprofilaxis (34\%). Si consideramos al grupo de pacientes con al menos uno de los factores de riesgo de TEV identificados en el análisis multivariable esta proporción aumenta a 52\% (40 de 76 
pacientes, Figura 1), poniendo de manifiesto el problema de la subutilización de esta importante medida preventiva. A pesar de lo llamativo de estas cifras, no son distintas a las reportadas internacionalmente. Una serie de estudios observacionales han revelado que actualmente sólo 40\%-50\% de los pacientes médicos de alto riesgo de TEV (incluyendo los pacientes con cáncer) reciben tromboprofilaxis farmacológica ${ }^{20-23}$.

En un estudio realizado en Brigham and Women's Hospital que analizó el efecto de la implementación de un sistema de alerta electrónico para el uso de tromboprofilaxis en pacientes médicos de alto riesgo ( $80 \%$ con cáncer), reveló que solamente en 13\% (163/1.251) de los pacientes se indicó tromboprofilaxis farmacológica antes de iniciar el estudio, proporción que aumentó a $23 \%$ (296/1.255) luego de iniciar las alertas. Este aumento fue suficiente para disminuir significativamente la frecuencia de TEV sintomático $(8,2 \%$ vs $4,9 \% ; \mathrm{p}=0,001)^{24}$.

El carácter retrospectivo de esta investigación no nos permite profundizar acerca de las causas de la subutilización de tromboprofilaxis farmacológica; solamente pudimos observar una pequeña proporción de pacientes con contraindicaciones al uso de anticoagulantes como causa de esta

\section{REFERENCIAS}

1. Geerts Wh, Pineo GF, Heit Ja, Bergevist D, Lassen MR, Colwell CW ET AL. Prevention of venous thromboembolism: the Seventh ACCP Conference on Antithrombotic and Thrombolytic Therapy. Chest 2004; 126 (3 Suppl): 338S-400S.

2. Alikhan R, Peters F, Wilmott R, Cohen AT. Fatal pulmonary embolism in hospitalized patients: a necropsy review. J Clin Pathol 2004; 57: 1254-7.

3. Baglin TP, White K, Charles A. Fatal pulmonary embolism in hospitalized medical patients. J Clin Pathol 1997; 50: 609-10.

4. Dentali F, Douketis JD, Gianni M, Lim W, Crowther MA. Meta-analysis: anticoagulant prophylaxis to prevent symptomatic venous thromboembolism in hospitalized medical patients. Ann Intern Med 2007; 146: 278-88.

5. Alikhan R, Cohen AT, Combe S, Samama MM, Desjardins L, ELDOR A ET AL. Risk factors for venous thromboem- situación (coagulopatía, trombocitopenia severa, hemorragia como motivo de ingreso).

El TEV es considerado actualmente un problema de salud pública que requiere la implementación urgente de medidas preventivas para disminuir su costo humano y económico ${ }^{25-27}$. Las consecuencias negativas no sólo resultan de los efectos del evento agudo (mortalidad, estadías hospitalarias prolongadas) sino también de complicaciones tardías como recurrencias ${ }^{28}$, hipertensión pulmonar ${ }^{29}$ y síndrome postrombótico ${ }^{30}$, que pueden afectar significativamente la calidad de vida de los pacientes.

Conclusiones: Los pacientes con cáncer hospitalizados por cuadros médicos agudos tienen un riesgo elevado de TEV sintomático (3\% en esta serie). Este riesgo disminuye significativamente con el uso de tromboprofilaxis farmacológica. Aunque se reconoce el alto riesgo de TEV en esta población de pacientes, una importante proporción no recibió tromboprofilaxis, fenómeno que ha sido reportado extensamente en recientes investigaciones.

La información revelada en este estudio indica la necesidad de implementar programas de educación y guías clínicas locales en cada institución con el fin de disminuir la frecuencia de esta importante pero prevenible complicación.

bolism in hospitalized patients with acute medical illness: analysis of the MEDENOX Study. Arch Intern Med 2004; 164: 963-8.

6. Goldhaber SZ, Dunn K, MacDougall RC. New onset of venous thromboembolism among hospitalized patients at Brigham and Women's Hospital is caused more often by prophylaxis failure than by withholding treatment. Chest 2000; 118: 1680-4.

7. Piazza G, Seddighzadeh A, Goldhaber SZ. Double trouble for 2,609 hospitalized medical patients who developed deep vein thrombosis: prophylaxis omitted more often and pulmonary embolism more frequent. Chest 2007; 132: 554-61.

8. Samama MM, Cohen AT, Darmon JY, Desjardins L, Eldor A, Janbon C ET AL. A comparison of enoxaparin with placebo for the prevention of venous thromboembolism in acutely ill medical patients. Prophylaxis in Medical Patients with Enoxaparin Study Group. N Engl J Med 1999; 341: 793-800.

9. Leizorovicz A, Cohen AT, Turpie AG, Olsson CG, 
VAitKus PT, Goldhaber SZ. Randomized, placebocontrolled trial of dalteparin for the prevention of venous thromboembolism in acutely ill medical patients. Circulation 2004; 110: 874-9.

10. Cohen AT, Davidson BL, Gallus AS, Lassen MR, Prins MH, Tomkowski W ET AL. Efficacy and safety of fondaparinux for the prevention of venous thromboembolism in older acute medical patients: randomized placebo controlled trial. BMJ 2006; 332: 325-9.

11. Stein PD, Beemath A, Meyers FA, Skaf E, Sánchez J, OLSON RE. Incidence of venous thromboembolism in patients hospitalized with cancer. Am J Med 2006; 119: 60-8.

12. Khorana AA, Francis CW, Culakova E, Fisher RI, Kuderer NM, Lyman GH. Thromboembolism in hospitalized neutropenic cancer patients. J Clin Oncol 2006; 24: 484-90.

13. Khorana AA, Francis CW, Culakova E, Kuderer NM, Lyman GH. Frequency, risk factors, and trends for venous thromboembolism among hospitalized cancer patients. Cancer 2007; 110: 2339-46.

14. Bergqvist D. Risk of venous thromboembolism in patients undergoing cancer surgery and options for thromboprophylaxis. J Surg Oncol 2007; 95: 167-74.

15. Alikhan R, Cohen AT, Combe S, Samama MM, Desjardins L, Eldor A ET AL. Prevention of venous thromboembolism in medical patients with enoxaparin: a subgroup analysis of the MEDENOX study. Blood Coagul Fibrinolysis 2003; 14: 341-6.

16. Kröger K, Weiland D, Ose C, Neumann N, Weiss S, HiRsCh C ET AL. Risk factors for venous thromboembolic events in cancer patients. Ann Oncol 2006; 17 : 297-303.

17. Otten HM, Mathijssen J, Ten Cate H, Soesan M, Inghels M, Richel DJ ET AL. Symptomatic venous thromboembolism in cancer patients treated with chemotherapy: an underestimated phenomenon. Arch Intern Med 2004; 164: 190-4.

18. Khorana AA, Francis CW, Culakova E, Lyman GH. Risk factors for chemotherapy-associated venous thromboembolism in a prospective observational study. Cancer 2005; 104: 2822-9.

19. Khorana AA, Francis CW, Culakova E. Thromboembolism is a leading cause of death in cancer patients receiving outpatient chemotherapy. J Thromb Haemost 2007; 5: 632-4.
20. Kahn SR, Panju A, Geerts W, Pineo GF, Desjardins L, Turpie AG et aL; CURVE study investigators. Multicenter evaluation of the use of venous thromboembolism prophylaxis in acutely ill medical patients in Canada. Thromb Res 2007; 119: 145-55.

21. Amin A, Stemkowski S, Lin J, Yang G. Thromboprophylaxis rates in US medical centers: success or failure? J Thromb Haemost 2007; 5: 1610-6.

22. Tapson VF, Decousus H, Pini M, Chong BH, Froehlich JB, Monreal M ET AL. IMPROVE Investigators. Venous thromboembolism prophylaxis in acutely ill hospitalized medical patients: findings from the International Medical Prevention Registry on Venous Thromboembolism. Chest 2007; 132: 936-45.

23. Cohen aT, Tapson VF, Bergmann JF, Goldhaber SZ, Kakkar AK, Deslandes B et al. Venous thromboembolism risk and prophylaxis in the acute hospital care setting (ENDORSE study): a multinational crosssectional study. Lancet 2008; 371: 387-94.

24. Kucher N, Koo S, Quiroz R, Cooper JM, Paterno MD, SoukonNikov B ET AL. Electronic alerts to prevent venous thromboembolism among hospitalized patients. N Engl J Med 2005; 352: 969-77.

25. Moll S, Mackman N. Venous Thromboembolism: A Need for More Public Awareness and Research Into Mechanisms. Arterioscler Thromb Vasc Biol 2008; 28: 367-9.

26. Cohen AT, Agnelli G, Anderson FA, Arcelus JI, Bergqvist D, Brecht JG et al; VTE Impact Assessment Group in Europe (VITAE). Venous thromboembolism (VTE) in Europe. The number of VTE events and associated morbidity and mortality. Thromb Haemost 2007; 98: 756-64.

27. MacDougall DA, Feliú AL, Boccuzzi SJ, Lin J. Economic burden of deep-vein thrombosis, pulmonary embolism, and post-thrombotic syndrome. Am J Health Syst Pharm 2006; 63(20 Suppl 6): S5-15.

28. Heit JA, Mohr DN, Silverstein MD, Petterson TM, O'Fallon WM, Melton JM. Predictors of recurrence after deep vein thrombosis and pulmonary embolism: a population-based cohort study. Arch Intern Med 2000; 160: 761-8.

29. Pengo V, Lensing AW, Prins MH, Marchiori A, Davidson BL, Tiozzo F ET AL. Incidence of chronic thromboembolic pulmonary hypertension after pulmonary embolism. N Engl J Med 2004; 350: 2257-64.

30. KAHN SR. The post-thrombotic syndrome: progress and pitfalls. Br J Haematol 2006; 134: 357-65. 